\title{
Effects of dabigatran regulates no-reflow phenomenon in acute myocardial infarction mice through anti-inflammatory and anti-oxidative activities and connective tissue growth factor expression
}

\author{
KUNQING SONG, YANSHAN WANG, JIZHANG SHENG, CHUNMEI MA and HONGMEI LI
}

Department of 4th Vasculocardiology, Cangzhou City Central Hospital, Cangzhou, Hebei 061000, P.R. China

Received June 25, 2016; Accepted June 19, 2017

DOI: $10.3892 / \mathrm{mmr} .2017 .7861$

\begin{abstract}
Pradaxa is a novel oral anticoagulant, which was originally used to prevent thrombosis following joint replacement surgery. The aim of the current study was to investigate the effect dabigatran on acute myocardial infarction through regulating no-reflow phenomenon and oxidative stress, neutrophil intraplaque infiltration and apoptosis. In the present study, dabigatran significantly inhibited the infarct size, increased arterial pressure and reduced no-reflow phenomenon in acute myocardial infarction (AMI) vehicle rabbits. Treatment with dabigatran significantly inhibited the P65 of nuclear factor $\kappa \mathrm{B}$, tumor necrosis factor $\alpha$, interleukin (IL) $-1 \beta$ and IL- 6 activities and significantly enhanced the catalase and superoxide dismutase activities in the AMI rabbits. In addition, dabigatran significantly suppressed inducible nitric oxide synthase (iNOS), collagen I, transforming growth factor $\beta 1$ (TGF- $\beta 1), \alpha$-smooth muscle actin ( $\alpha$-SMA) and connective tissue growth factor (CTGF) protein expression in AMI rabbits. Taken together, these results suggest that the effects of dabigatran inhibit no-reflow phenomenon, infarct size and enhance arterial pressure in AMI through anti-inflammatory and anti-oxidative activity, and regulating iNOS, collagen I, TGF- $\beta 1, \alpha-S M A$ and CTGF protein expression in AMI rabbits.
\end{abstract}

\section{Introduction}

Myocardial infarction (MI), commonly known as a heart attack, occurs when blood flow stops to a part of the heart, causing damage to the heart muscle (1). Common symptoms are chest pain and acute circulatory dysfunction (2). According to clinical processes and electrocardiographic results,

Correspondence to: Mr. Kunqing Song, Department of 4th Vasculocardiology, Cangzhou City Central Hospital, 201 Xinhua Road, Cangzhou, Hebei 061000, P.R. China

E-mail: suhuaichengqiao@126.com

Key words: dabigatran, no-reflow phenomenon, acute myocardial infarction, inflammation, oxidative myocardial infarction is classified into three phases; acute, subacute and chronic. Its clinical symptoms predominantly appear during the acute stage (3).

Acute MI(AMI) is a serious type of coronary heart disease (4). AMI as a result of coronary artery disease occurs when the blood supply of coronary artery reduces sharply or suspends completely, resulting in lasting and severe acute ischemia of the corresponding myocardium (5). Therefore, myocardial ischemic necrosis of this myocardium occurs.

In 2008, as a non-pseudo-peptide thrombin inhibitor, Pradaxa, which was developed by Boehringer Ingelheim, came into the market in Germany and the UK as a novel oral anticoagulant (6). In October 2010, it was approved by Food and Drug Administration to be employed to prevent cerebral apoplexy for patients with auricular fibrillation (7). Pradaxa is effective to reduce risks of patients with atrial fibrillation (8). In addition, it can avoid the reinforcement of intracranial hemorrhage (9). It is preferable than Warfarin and provides a new choice for anticoagulant therapy of atrial fibrillation (10). In the present study it was investigated whether effects of dabigatran regulate the no-reflow phenomenon, inflammation and oxidative stress in AMI rabbits.

\section{Materials and methods}

Surgical and experimental procedures. Male New Zealand White rabbits (2.3-3.0 kg, 4-5 months age, $\mathrm{n}=24)$ were purchased from the Experimental Center of Hebel Medical University (Hebel, China) and housed at $22-24^{\circ} \mathrm{C}$ with a $12-\mathrm{h}$ light/dark cycle in 55-60\% humidity with access to food and water. All rabbits were used as AMI rabbits and anesthetized with ketamine $(75 \mathrm{mg} / \mathrm{kg})$ and xylazine $(5 \mathrm{mg} / \mathrm{kg})$. Rabbits were ventilated mechanically following intubation and its catheters were inserted into the jugular vein, carotid artery and left atrial appendage. The heart rate and arterial blood pressure were monitored and recorded. The coronary artery was occluded for 30 min following a stabilization period, then were reperfused for $3 \mathrm{~h}$. Blue pigment was injected with the re-occlusion of the coronary artery, which set the limits of the ischemic risk zone. Zones of necrosis stained with triphenyltetrazolium chloride were distinguished. The anatomic no-reflow zone was measured using thioflavin $\mathrm{S}$ 
(Sigma-Aldrich; Merck KGaA, Darmstadt, Germany). Intact endothelium stained with fluorescent yellow dye served as a marker of regional perfusion. Areas receiving blood flow appear brightly fluorescent viewed under ultraviolet light, and the no-reflow zone appears as a nonfluorescent, dark area. Experiments were approved by the Medical Ethics Committee of Cangzhou City Central Hospital (Cangzhou, China).

Treatment groups. All rabbits were randomly distributed into the control, AMI vehicle and dabigatran group. In the control or AMI vehicle groups, normal rabbits and AMI model rabbits received an equivalent volume of vehicle. In the dabigatran group, AMI model rabbits were administered as an intravenous bolus $(0.5 \mathrm{mg} / \mathrm{kg})$ and concomitant infusion $(0.15 \mathrm{mg} / \mathrm{kg} / \mathrm{h})$. Prior to treatment initiation, the coronary artery was occluded for $15 \mathrm{~min}$ and then the infusion was continued for $2.5 \mathrm{~h}$ during reperfusion.

Inflammation and oxidative stress measurements. Blood samples were obtained at baseline, then and centrifuged at $2,000 \mathrm{x} \mathrm{g}$ for $10 \mathrm{~min}$ at $4^{\circ} \mathrm{C}$. After centrifugation, the plasma was removed and the samples stored at $-70^{\circ} \mathrm{C}$ until required for analysis. The plasma was used to analyze the P65 of nuclear factor (NF)- $\mathrm{B}$ (H202), tumor necrosis factor (TNF)- $\alpha$ (H052), interleukin (IL)-1 $\beta$ (H002), IL-6 (H007), catalase (CAT; A007-1-1) and superoxide dismutase (SOD; A001-1-1) activities using ELISA kits (Nanjing Jiancheng Bioengineering Institute, Nanjing, China) in accordance with the manufacturer's instructions.

Western blotting. Heart samples were obtained at baseline and were homogenated using a radioimmunoprecipitation acid lysis buffer (Beyotime Institute of Biotechnology, Haimen, China). Following centrifugation at $12,000 \mathrm{x}$ g for $10 \mathrm{~min}$ at $4^{\circ} \mathrm{C}$, supernatant liquor was collected to quantify protein content using a Bicinchoninic Acid Assay kit (Beyotime Institute of Biotechnology). Proteins $(50 \mu \mathrm{g})$ were separated on a $10-12 \%$ SDS polyacrylamide gel and transferred to a polyvinylidene difluoride membrane (Beyotime Institute of Biotechnology). The membranes were incubated overnight at $4^{\circ} \mathrm{C}$ with anti-inducible nitric oxide synthase (iNOS; cat. no. sc-649; 1:500; Santa Cruz Biotechnology, Inc., Dallas, TX, USA), anti-collagen I (cat.no. 84336; 1:500; Cell Signaling Technology, Inc., Danvers, MA, USA), anti-transforming growth factor $\beta 1$ (TGF- $\beta 1$; cat. no. sc-9043; Santa Cruz Biotechnology, Inc.), anti- $\alpha$-smooth muscle actin ( $\alpha$-SMA; cat. no. 19245 ; $1: 500$; Santa Cruz Biotechnology, Inc.), anti-connective tissue grow th factor (CTGF; cat. no. sc-25440; 1:500; Santa Cruz Biotechnology, Inc.) and anti- $\beta$-actin (cat. no. sc-7210; 1:500; Santa Cruz Biotechnology, Inc.) antibodies after membrane incubation in 5\% skimmed milk powder. Membranes were then washed twice with TBS with $0.1 \%$ Tween-20 and incubated for $1 \mathrm{~h}$ with the goat anti-rabbit peroxidase-conjugated secondary antibody (cat. no. sc-2004; 1:5,000; Santa Cruz Biotechnology, Inc.) at $37^{\circ} \mathrm{C}$.

Statistical analysis. Data are presented as the mean and standard deviation using SPSS version 17.0 software (SPSS, Inc., Chicago, IL, USA) Statistical analysis was conducted using a one-way analysis of variance. $\mathrm{P}<0.05$ was considered to indicate a statistically significant difference.

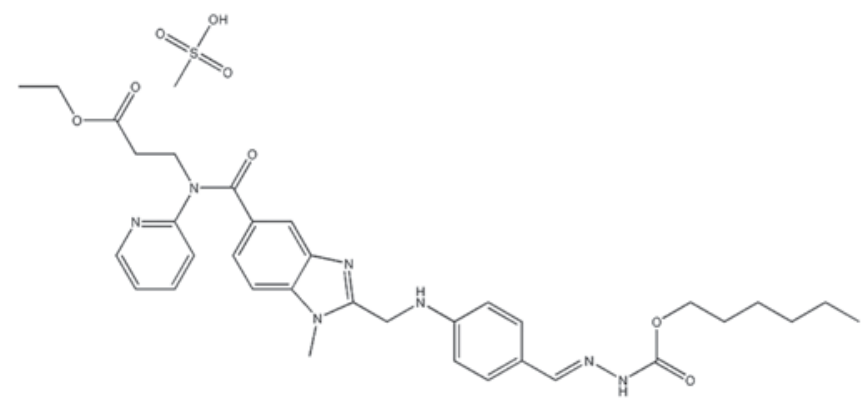

Figure 1. The chemical structure of dabigatran.
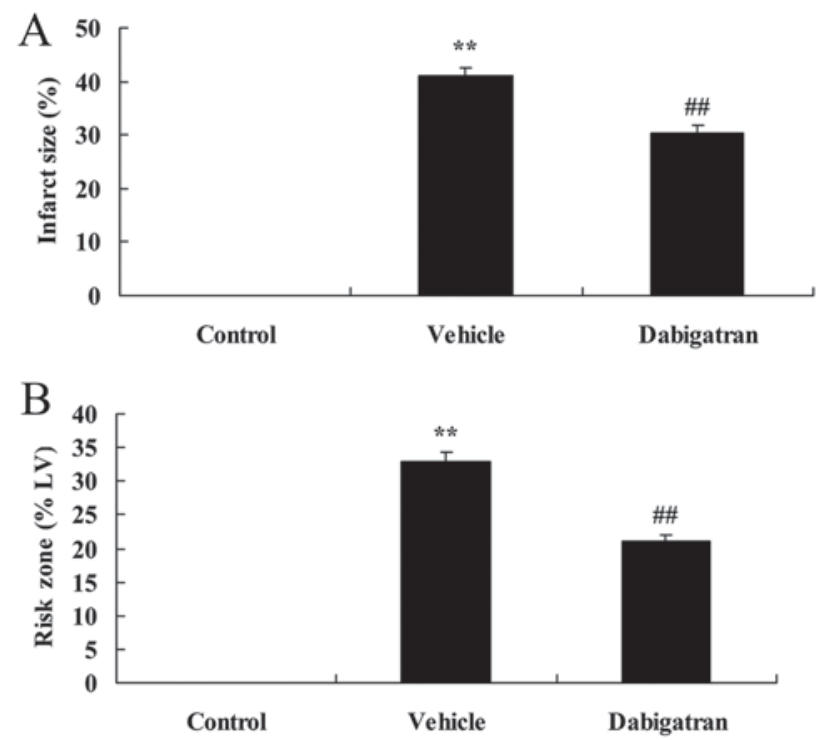

Figure 2. Effects of dabigatran on infarct size in AMI rabbits. Effects of dabigatran on (A) infarct size and (B) risk zone of infarct size in AMI rabbits. ${ }^{* *} \mathrm{P}<0.01$ vs. control group; ${ }^{\# \prime} \mathrm{P}<0.01$ vs. vehicle group. AMI, acute myocardial infarction.

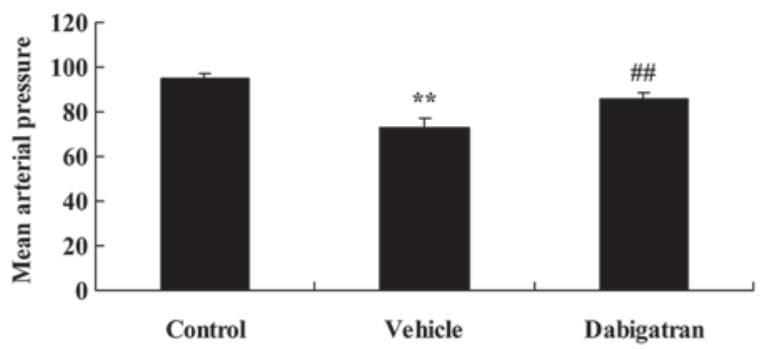

Figure 3. Effects of dabigatran on arterial pressure in acute myocardial infarction rabbits. ${ }^{* *} \mathrm{P}<0.01$ vs. control group; ${ }^{\# \#} \mathrm{P}<0.01$ vs. vehicle group.

\section{Results}

Effects of dabigatran on infarct size in AMI rabbits. The chemical structure of dabigatran is presented in Fig. 1. In order to examine the effects of dabigatran on infarct size in AMI rabbits, the AMI model of rabbits was used. The results of Fig. 2 indicate that infarct size and risk zone of infarct size in the AMI vehicle group were higher than that of the control group. In addition, the effect of dabigatran significantly inhibited the infarct size and risk zone of infarct size in AMI rabbits, compared with AMI vehicle group (Fig. 2). 

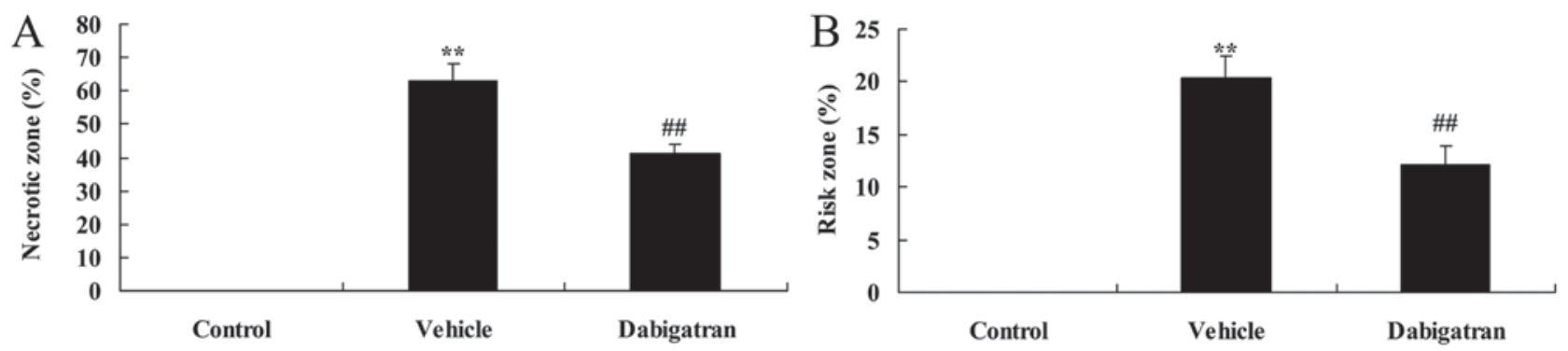

Figure 4. Effects of dabigatran on no-reflow phenomenon in AMI rabbits. Effects of dabigatran on (A) necrotic zone and (B) risk zone of necrotic zone in AMI rabbits. ${ }^{* *} \mathrm{P}<0.01$ vs. control group; ${ }^{\#} \mathrm{P}<0.01$ vs. vehicle group. $\mathrm{AMI}$, acute myocardial infarction.
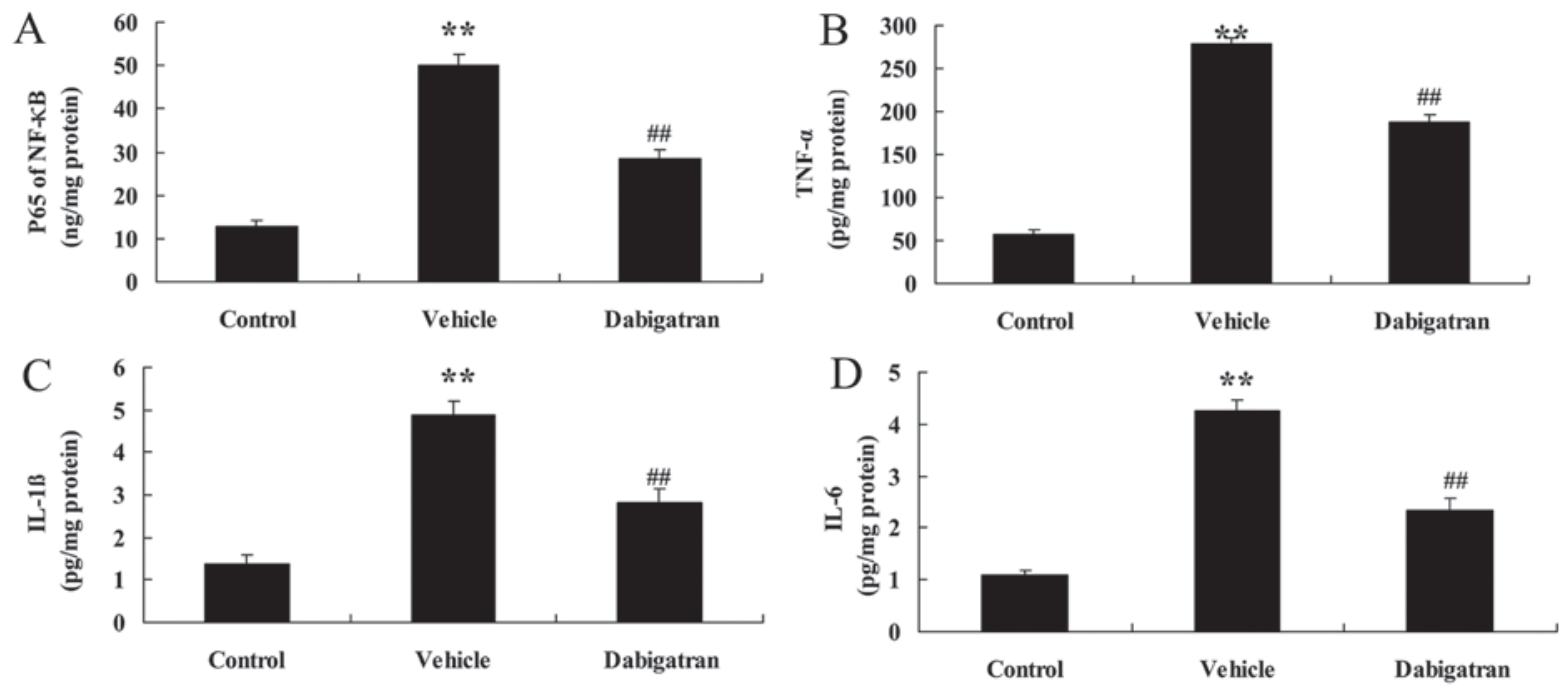

Figure 5. Effects of dabigatran on inflammation in AMI rabbits. Effects of dabigatran on P65 of (A) NF-kB, (B) TNF- $\alpha$, (C) IL-1 $\beta$ and (D) IL-6 activities in $A M I$ rabbits. ${ }^{* *} \mathrm{P}<0.01$ vs. control group; ${ }^{\# \#} \mathrm{P}<0.01$ vs. vehicle group. AMI, acute myocardial infarction; NF- $\mathrm{kB}$, nuclear factor $\kappa \mathrm{B}$; TNF- $\alpha$, tumor necrosis factor $\alpha$; IL-1 $\beta$, interleukin $1 \beta$.

Effects of dabigatran on arterial pressure in AMI rabbits. Subsequently, the effects of dabigatran on arterial pressure in AMI rabbits were investigated. As presented in Fig. 3, AMI reduced arterial pressure in AMI rabbits, compared with control rabbits. However, the effect of dabigatran significantly increased arterial pressure in AMI rabbits, compared with AMI vehicle rabbits (Fig. 3).

Effects of dabigatran on no-reflow phenomenon in AMI rabbits. To examine the effects of dabigatran on arterial pressure in AMI rabbits, no-reflow phenomenon was measured in the current study. Fig. 4 indicates that no-reflow phenomenon was higher in the vehicle group compared with that of the control group. The effect of dabigatran significantly inhibited the AMI-induced no-reflow phenomenon, compared with AMI vehicle rabbits (Fig. 4).

Effects of dabigatran on inflammation in AMI rabbits. The effects of dabigatran on arterial pressure in AMI rabbits were established. There was a significant increase in the activities of P65 of NF- $\kappa$ B, TNF- $\alpha$, IL- $1 \beta$ and IL-6 in AMI rabbits, compared with the normal control group (Fig. 5). Inhibition of the P65 of NF- $\kappa$ B, TNF- $\alpha$, IL-1 $\beta$ and IL- 6 activities was observed in the dabigatran group, compared with that of AMI rabbits (Fig. 5).
Effects of dabigatran on oxidative stress in AMI rabbits. To investigate the effects of dabigatran on oxidative stress in AMI rabbits, CAT and SOD activities were detected using ELISA kits. Compared with normal control group, the CAT and SOD activities were significantly inhibited in the AMI rabbits group (Fig. 6). The effect of dabigatran significantly enhanced the CAT and SOD activities in the AMI rabbits, compared with AMI rabbits group (Fig. 6).

Effects of dabigatran on iNOS and collagen I in AMI rabbits. To examine the effects of dabigatran on iNOS and collagen I protein expression in AMI rabbits, we detected the expression of iNOS and collagen I using western blotting. As presented in Fig. 7, AMI significantly activated the iNOS and collagen I protein expression in rabbits, compared with the normal control group. However, the effect of dabigatran significantly suppressed the iNOS and collagen I protein expression in AMI rabbits, compared with AMI rabbits group (Fig. 7).

Effects of dabigatran on TGF- $\beta 1, \alpha-S M A$ and CTGF in AMI rabbits. To further confirm the role of TGF- $\beta 1, \alpha-S M A$ and CTGF in the effects of dabigatran on AMI, TGF- $\beta 1$ was measured using western blotting. As presented in Fig. 8, there a significant increase in TGF- $\beta 1, \alpha$-SMA and CTGF protein expression in AMI rabbits, compared with normal 

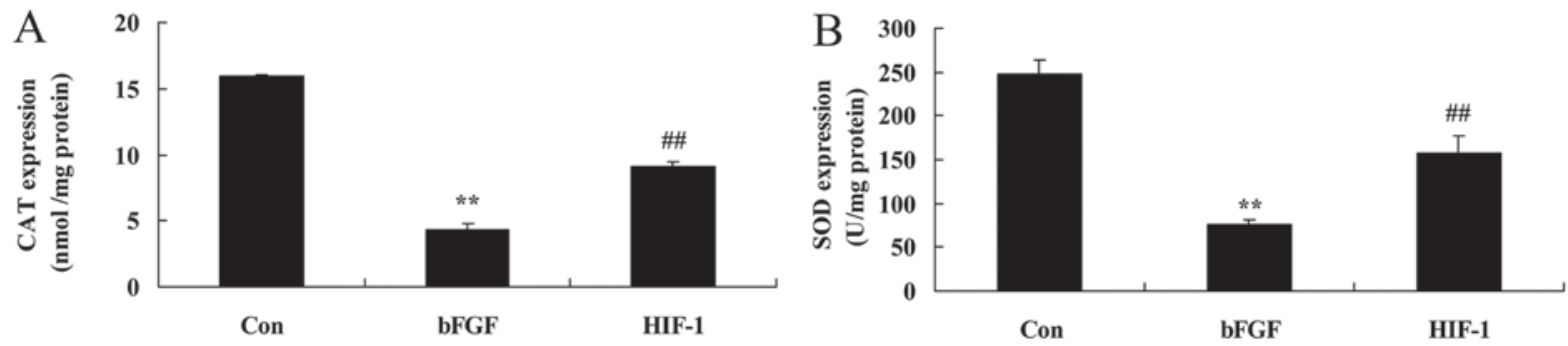

Figure 6. Effects of dabigatran on oxidative stress in AMI rabbits. Effects of dabigatran on (A) CAT and (B) SOD activities in AMI rabbits. * P $<0.01$ vs. control group; ${ }^{\# \#} \mathrm{P}<0.01$ vs. vehicle group. AMI, acute myocardial infarction; CAT, catalase; SOD, superoxide dismutase.

A

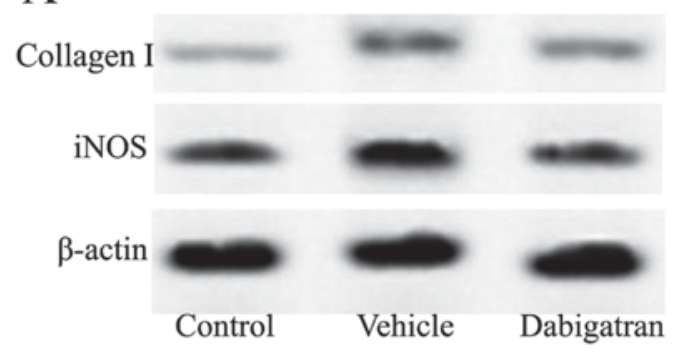

$\mathrm{B}$

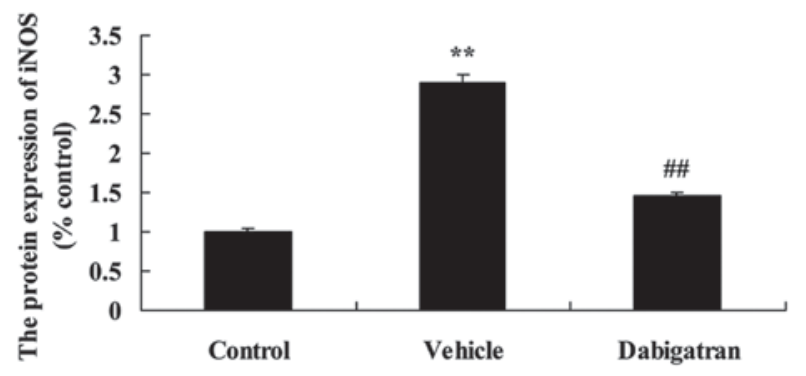

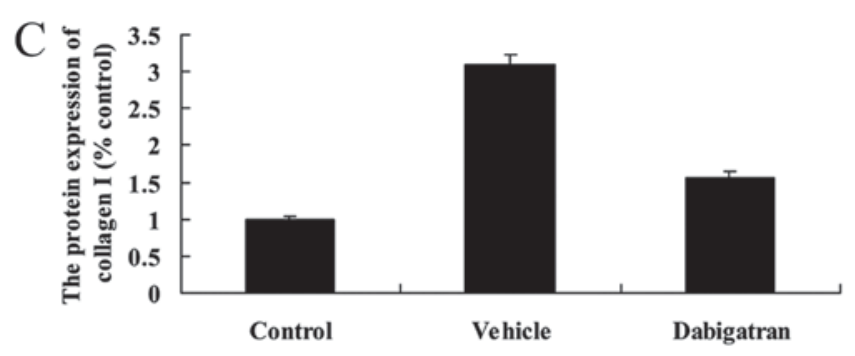

Figure 7. Effects of dabigatran on iNOS and collagen I in AMI rabbits. Effects of dabigatran on iNOS and collagen I protein expression using (A) western blotting and statistical analysis of (B) iNOS and (C) collagen I protein expression in AMI rabbits. ${ }^{* *} \mathrm{P}<0.01$ vs. control group; ${ }^{\# \#} \mathrm{P}<0.01$ vs. vehicle group. iNOS, nitric oxide synthase; AMI, acute myocardial infarction.

A
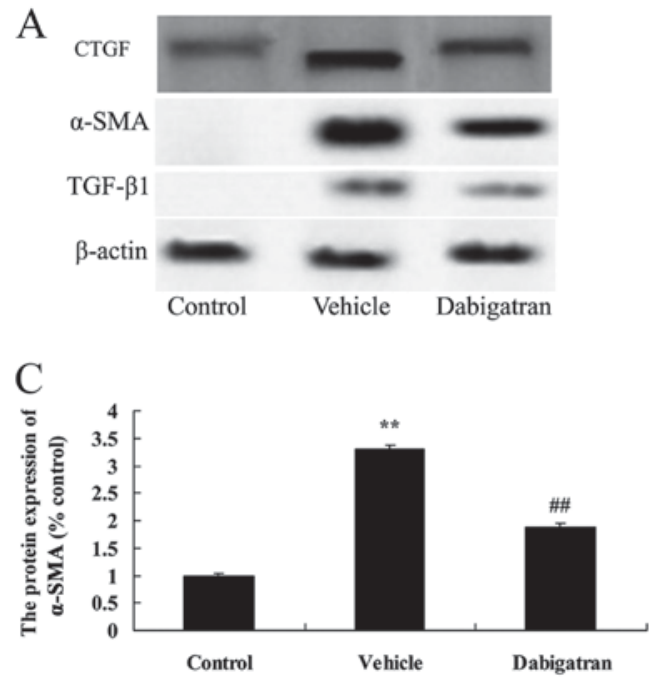

$\mathrm{B}$

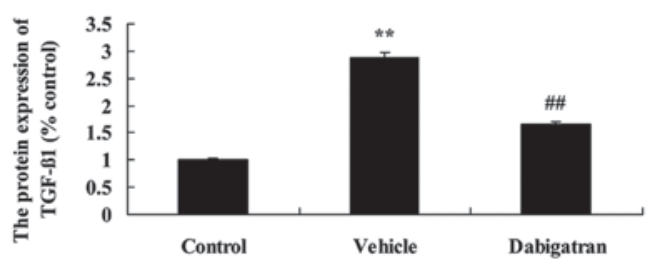

$\mathrm{D}$

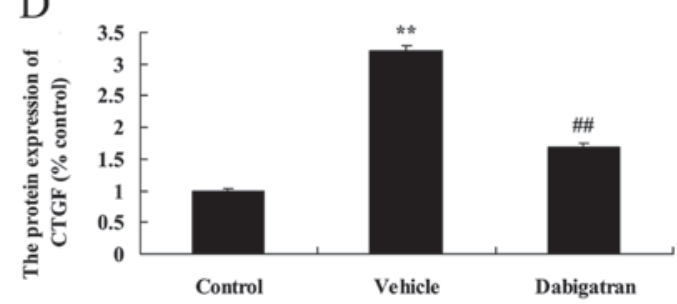

Figure 8. Effects of dabigatran on TGF- $\beta 1, \alpha$-SMA and CTGF in AMI rabbits. Effects of dabigatran on TGF- $\beta 1, \alpha$-SMA and CTGF protein expression using (A) western blotting and statistical analysis of (B) TGF- $\beta 1$, (C) $\alpha$-SMA and (D) CTGF protein expression in AMI rabbits. ${ }^{* *} \mathrm{P}<0.01$ vs. control group; ${ }^{\# \#} \mathrm{P}<0.01$ vs. vehicle group. TGF- $\beta 1$, transforming growth factor $\beta 1$; AMI, acute myocardial infarction.

control group. However, the effects of dabigatran significantly suppressed the TGF- $\beta 1, \alpha$-SMA and CTGF protein expression in AMI rabbits, compared with AMI rabbits group (Fig. 8). 


\section{Discussion}

AMI is an acute event caused by atheromatous thrombosis and is a major cause of angiocardiopathic mortality (11). The most effective treatment for myocardial ischemic injury is to recover the blood supply as quickly as possible $(11,12)$. However, studies have identified that following a period of ischemia, myocardial injuries worsen upon reperfusion; reversible injuries of cardiomyocytes can become irreversible. Myocardial ischemia/reperfusion injuries reduced cardiac function and malignant arrhythmia (13). An AMI rabbit model was used to verify that dabigatran significantly inhibited the infarct size and increased arterial pressure in AMI vehicle rabbits.

Lesion plaques of AMI patients easily rupture and are unstable, resulting in acute and complete or incomplete occlusive thrombus lesions of the coronary arteries (14). A previous study suggested that the local inflammatory response could trigger atherosclerosis, resulting in insatiability of plaques that are easy to rupture (15). A literature review indicated that following AMI, overexpression of IL may occur, which suggested that inflammation additionally participates in the occurrence of AMI (14). The concentration of inflammatory cytokines in the serum is associated with the severity of the disease. In the present study, dabigatran significantly reduced the no-reflow phenomenon in AMI vehicle rabbits.

Atherosclerosis is a pathogenesis basis of coronary heart disease, while inflammation is an important feature of atherosclerosis (16). With clear biological effects, inflammatory factors are a type of endogenous polypeptide produced by immunocytes (17). They can mediate various immune responses and are closely associated with occurrence and development of atherosclerosis (18). IL-6 is a type of inflammatory cytokine with multiple functions, which is secreted by active monocytes, macrophages, $\mathrm{T}$ lymphocytes, endothelial cells and fibroblasts (19). IL-6 has been reported to be the most powerful predictive factor of heart damage and mortality for patients with AMI (20). TNF- $\alpha$ is a multi-functional protein predominantly produced by active macrophage/monocyte system. Under physiological states, myocardial cells do not generate TNF- $\alpha$. However, when myocardial infarction-induced pump failure occurs, expression of TNF- $\alpha$ greatly increases, thus is a reliable indicator for clinical prognosis of AMI (21). In the current study, it was identified that dabigatran significantly inhibited P65 of NF- $\kappa \mathrm{B}$, TNF- $\alpha$, IL- $1 \beta$ and IL-6 activities and significantly enhanced CAT and SOD activities in the AMI rabbits. De Boer et al (10) reported that the effect of dabigatran inhibits allergic lung inflammation through suppression of inflammation.

As a cellular messenger modulating functions of the cardiovascular system, nervous system and immune system, NO is a biological information transmitter (22). Numerous studies have observed that NO possesses anti-platelet aggregation, inhibition of hyperplasia of the vascular smooth muscle, interaction between endothelial cells, regulation of angiostasis and mediation of immune and cytotoxic effects (23). AMI may serve an essential role in cardiovascular diseases, such as hypertension (24). Previously, a study identified that dabigatran significantly suppressed the iNOS, collagen I, TGF- $\beta 1$, $\alpha$-SMA and CTGF protein expression in AMI rabbits. Bogatkevich et al $(25,26)$ reported that dabigatran demonstrates antifibrotic effects on lung fibroblasts through $\alpha$-SMA and type I collagen expression and dabigatran etexilate in a murine model of interstitial lung disease through anti-inflammatory effects and TGF- $\beta$.

In conclusion, the effects of dabigatran on infarct size, arterial pressure and no-reflow phenomenon, were observed to include anti-inflammatory and anti-oxidative effects. The effects of dabigatran may be mediated through downregulation of iNOS, collagenI, TGF- $\beta 1, \alpha-$ SMA and CTGFexpression in AMI rabbits. There is a requirement to conduct clinical trials to fully assess the effects of dabigatran no-reflow phenomenon in AMI rabbits and assess the risks involved in longterm therapy with this novel oral anticoagulant agent.

\section{References}

1. Gili M, Ramirez G, Béjar L and López J: Is cocaine-associated acute myocardial infarction the same as myocardial infarction associated with recent cocaine consumption? Response. Rev Esp Cardiol (Eng1 Ed) 67: 965-966, 2014.

2. Kidawa M, Chizyński K, Zielińska M, Kasprzak JD and Krzeminska-Pakula M: Real-time 3D echocardiography and tissue Doppler echocardiography in the assessment of right ventricle systolic function in patients with right ventricular myocardial infarction. Eur Heart J Cardiovasc Imaging 14: 1002-1009, 2013

3. Kolte D, Khera S, Aronow WS, Mujib M, Palaniswamy C, Sule S, Jain D, Gotsis W, Ahmed A, Frishman WH and Fonarow GC: Trends in incidence, management, and outcomes of cardiogenic shock complicating ST-elevation myocardial infarction in the United States. J Am Heart Assoc 3: e000590, 2014.

4. Sondergaard CS, Hess DA, Maxwell DJ, Weinheimer C, Rosová I, Creer MH, Piwnica-Worms D, Kovacs A, Pedersen L and Nolta JA: Human cord blood progenitors with high aldehyde dehydrogenase activity improve vascular density in a model of acute myocardial infarction. J Transl Med 8: 24, 2010.

5. Malik MA, Alam Khan S, Safdar S and Taseer IU: Chest Pain as a presenting complaint in patients with acute myocardial infarction (AMI). Pak J Med Sci 29: 565-568, 2013.

6. Dzeshka MS and Lip GY: Warfarin versus dabigatran etexilate: An assessment of efficacy and safety in patients with atrial fibrillation. Expert Opin Drug Saf 14: 45-62, 2015.

7. Grottke O, van Ryn J, Spronk HM and Rossaint R: Prothrombin complex concentrates and a specific antidote to dabigatran are effective ex-vivo in reversing the effects of dabigatran in an anticoagulation/liver trauma experimental model. Crit Care 18: R27, 2014.

8. Sanabria C, Cabrejos J, Olortegui A, Guevara C and Garrido Lecca S: Costo-efectividad De apixaban con otros noacs (Dabigatran Y Rivaroxaban) En El tratamiento De La fibrilacion auricular no valvular (Fanv) En pacientes De La seguridad social De Perú. Value Health 18: A829, 2015.

9. Mohd Hajiri M, Shaharuddin S, Long CM, Hashim R, Zulkifly HH, Kasim SS and Lim CW: Preliminary study of safety and efficacy of warfarin versus dabigatran in atrial fibrillation patients in a tertiary hospital in Malaysia. Value Health 18: A378, 2015.

10. de Boer JD, Berkhout LC, de Stoppelaar SF, Yang J, Ottenhoff R, Meijers JC, Roelofs JJ, van't Veer C and van der Poll T: Effect of the oral thrombin inhibitor dabigatran on allergic lung inflammation induced by repeated house dust mite administration in mice. Am J Physiol Lung Cell Mol Physiol 309: L768-L775, 2015.

11. Feistritzer HJ, Klug G, Reinstadler SJ, Mair J, Seidner B, Mayr A, Franz WM and Metzler B: Aortic stiffness is associated with elevated high-sensitivity cardiac troponin $\mathrm{T}$ concentrations at a chronic stage after ST-segment elevation myocardial infarction. J Hypertens 33: 1970-1976, 2015.

12. Lax A, Sanchez-Mas J, Asensio-Lopez MC, Fernandez-Del Palacio MJ, Caballero L, Garrido IP, Pastor-Perez FJ, Januzzi JL and Pascual-Figal DA: Mineralocorticoid receptor antagonists modulate galectin-3 and interleukin-33/ST2 signaling in left ventricular systolic dysfunction after acute myocardial infarction. JACC Heart Fail 3: 50-58, 2015. 
13. Studer M, Zuber M, Jamshidi P, Buser P and Erne P: Thromboembolic acute myocardial infarction in a congenital double chambered left ventricle. Indian Heart J 63: 289-290, 2011.

14. Seropian IM, Cerliani JP, Toldo S, Van Tassell BW, Ilarregui JM, González GE, Matoso M, Salloum FN, Melchior R, Gelpi RJ, et al: Galectin-1 controls cardiac inflammation and ventricular remodeling during acute myocardial infarction. Am J Pathol 182: 29-40,2013.

15. Brener SJ, Cristea E, Kirtane AJ, McEntegart MB, Xu K, Mehran R and Stone GW: Intra-procedural stent thrombosis: A new risk factor for adverse outcomes in patients undergoing percutaneous coronary intervention for acute coronary syndromes JACC Cardiovasc Interv 6: 36-43, 2013.

16. Exaire JE, Fathi RB, Brener SJ, Karha J, Ellis SG and Bhatt DL: Impaired myocardial perfusion score and inflammatory markers in patients undergoing primary angioplasty for acute myocardial infarction. Arch Cardiol Mex 76: 376-382, 2006.

17. Hiroi T, Wajima T, Negoro T, Ishii M, Nakano Y, Kiuchi Y, Mori Y and Shimizu S: Neutrophil TRPM2 channels are implicated in the exacerbation of myocardial ischaemia/reperfusion injury. Cardiovasc Res 97: 271-281, 2013.

18. Mohseni M, Vafa M, Zarrati M, Shidfar F, Hajimiresmail SJ and Rahimi Forushani A: Beneficial effects of coenzyme Q10 supplementation on lipid profile and intereukin-6 and intercellular adhesion molecule-1 reduction, preliminary results of a double-blind trial in acute myocardial infarction. Int $\mathbf{J}$ Prev Med 6: 73, 2015

19. Lu W, Tang Y, Zhang Z, Zhang X, Yao Y, Fu C, Wang X and Ma G: Inhibiting the mobilization of Ly6C(high) monocytes after acute myocardial infarction enhances the efficiency of mesenchymal stromal cell transplantation and curbs myocardial remodeling. Am J Transl Res 7: 587-597, 2015.
20. Kretzschmar D, Betge S, Windisch A, Pistulli R, Rohm I, Fritzenwanger M, Jung C, Schubert K, Theis B, Petersen I, et al: Recruitment of circulating dendritic cell precursors into the infarcted myocardium and pro-inflammatory response in acute myocardial infarction. Clin Sci (Lond) 123: 387-398, 2012.

21. Rodriguez AE, Fernandez M, Santaera O, Larribau M, Bernardi V, Castaño H and Palacios LF: Coronary stenting in patients undergoing percutaneous transluminal coronary angioplasty during acute myocardial infarction. Am J Cardiol 77: 685-689, 1996 .

22. Aygül N, Aygül MU, Ozdemir K and Altunkeser BB: Emergency revascularization procedures in patients with acute ST-elevation myocardial infarction due to acute total occlusion of unprotected left main coronary artery: A report of five cases. Turk Kardiyol Dern Ars 38: 131-134, 2010.

23. Ahmed RP, Haider KH, Shujia J, Afzal MR and Ashraf M: Sonic Hedgehog gene delivery to the rodent heart promotes angiogenesis via iNOS/netrin-1/PKC pathway. PLoS One 5: e8576, 2010.

24. Ribeiro DA, Buttros JB, Oshima CT, Bergamaschi CT and Campos RR: Ascorbic acid prevents acute myocardial infarction induced by isoproterenol in rats: Role of inducible nitric oxide synthase production. J Mol Histol 40: 99-105, 2009.

25. Bogatkevich GS, Ludwicka-Bradley A and Silver RM: Dabigatran, a direct thrombin inhibitor, demonstrates antifibrotic effects on lung fibroblasts. Arthritis Rheum 60: 3455-3464, 2009.

26. Bogatkevich GS, Ludwicka-Bradley A, Nietert PJ, Akter T, van Ryn J and Silver RM: Antiinflammatory and antifibrotic effects of the oral direct thrombin inhibitor dabigatran etexilate in a murine model of interstitial lung disease. Arthritis Rheum 63: $1416-1425,2011$ 